\title{
La cultura del emprendimiento de Ecuador en relación a Perú
}

\author{
The culture of entrepreneurship in Ecuador in relation to Peru \\ Marcelo Fernando Villalba Díaz. ${ }^{1}$
}

\begin{abstract}
.
DOI: https://doi.org/10.33262/visionariodigital.v4i3.1340

En la actualidad, el emprendimiento es considerado como uno de los motores de crecimiento económico en los países, la relevancia que ha tomado el desarrollo de los emprendedores hace que el gobierno re direccione las políticas hacia el incentivo del emprendimiento innovador, también la empresa privada ha tenido iniciativas, y la propia sociedad les felicita por sus aportes ya que esta situación era muy diferente hace poco más de una década y media, la dimensión de la actividad emprendedora era apenas conocida, las iniciativas se enfocaban principalmente en las pymes, y la percepción sobre los emprendedores no era alentadora por la ausencia de datos, así como la falta de variedad de fuentes de información, lo que hacía difícil la comparación con otros países, es por ello que el centro de Desarrollo Emprendedor de la Universidad ESAN junto con el proyecto Global Entrepreneurship Monitor (GEM) en el año 2003 se unieron para impulsar la producción de conocimiento que potencie el emprendimiento, contribuya a la renovación del sector empresarial y fomente el desarrollo a partir de la innovación esto contribuye como una herramienta valiosa para desarrollar iniciativas que promuevan la actividad emprendedora y que esta genere un alto impacto $\mathrm{y}$ rendimiento en la sociedad.
\end{abstract}

Keywords: entrepreneurship, yield, GEM, TEA.

\footnotetext{
${ }^{1}$ Escuela Superior Politécnica de Chimborazo, Riobamba, Ecuador, marcelo.villalbad@espoch.edu.ec
} 


\section{Resumen.}

En la actualidad, el emprendimiento es considerado como uno de los motores de crecimiento económico en los países, la relevancia que ha tomado el desarrollo de los emprendedores hace que el gobierno re direccione las políticas hacia el incentivo del emprendimiento innovador, también la empresa privada ha tenido iniciativas, y la propia sociedad les felicita por sus aportes ya que esta situación era muy diferente hace poco más de una década y media, la dimensión de la actividad emprendedora era apenas conocida, las iniciativas se enfocaban principalmente en las pymes, y la percepción sobre los emprendedores no era alentadora por la ausencia de datos, así como la falta de variedad de fuentes de información, lo que hacía difícil la comparación con otros países, es por ello que el centro de Desarrollo Emprendedor de la Universidad ESAN junto con el proyecto Global Entrepreneurship Monitor (GEM) en el año 2003 se unieron para impulsar la producción de conocimiento que potencie el emprendimiento, contribuya a la renovación del sector empresarial y fomente el desarrollo a partir de la innovación esto contribuye como una herramienta valiosa para desarrollar iniciativas que promuevan la actividad emprendedora y que esta genere un alto impacto y rendimiento en la sociedad.

Palabras claves: emprendimiento, rendimiento, GEM, TEA.

\section{Introducción.}

El GEM fue creado con la finalidad de brindar información verdadera y comparable que permita realizar una comparación compleja entre el emprendimiento y el crecimiento económico, asimismo, el proyecto busca fomentar la creación de políticas basadas en hechos que mejoren la actividad emprendedora en los países del mundo, entonces el modelo es de tipo conceptual que relaciona directamente el emprendimiento con los mecanismos que afectan el crecimiento económico de un país por lo tanto el modelo cuenta con tres principios fundamentales: primero, se considera al emprendimiento como un proceso que se desarrolla dentro de un contexto ya sea político, social e histórico; segundo, reconoce que este proceso es desarrollado por personas con actitudes y aspiraciones; y, tercero, explica algunas de las razones por las cuales algunas economías se desarrollan más rápido que otras.

Para esto es necesario conocer cómo funciona el modelo emprendedor, todo comienza con una oportunidad o una descontinuación de los negocios esto nos da un emprendedor potencial cuando el negocio se está formando dicho emprendedor pasa de ser potencial a ser naciente esto aproximadamente puede durar de 3 a 42 meses, una vez que el negocio se gestiona esto puede durar de 42 meses en adelante hasta aproximadamente unos 5 años donde al emprendedor ya se lo considera como establecido y hasta ahí el proceso hasta que vuelva a empezar el ciclo. 
Para que esto sea posible también se necesita conocer las fases del desarrollo económico, se puede encontrar la economía que se basa en recursos naturales, negocios extractivos y fuerte dependencia de mano de obra, también podemos encontrar economía basadas en eficiencia con un crecimiento de la industrialización y las economías de escala con una marcada cadena de suministro y por otro lado se tiene la economía basada en innovación que pretende expandir el sector de servicios y aumentar el conocimiento. En los años 2016 y 2017 participaron 65 países y dentro del contexto Latinoamericano vemos que Ecuador y Perú se ubican en una economía basada en eficiencia si bien es cierto ya no es una economía basada en recursos, pero tampoco es una economía basada en innovación que sería el punto óptimo.

Estos resultados han potenciado las acciones de los demás integrantes que conforman el ecosistema emprendedor como la empresa privada, las organizaciones sin fines de lucro, la academia e, inclusive, la sociedad en su conjunto, se espera que este nuevo reporte continúe contribuyendo al debate y la formulación de políticas que promuevan el emprendimiento y la iniciativa para ello Global Entrepreneurship Monitor (GEM) es considerado como la mayor iniciativa de investigación sobre las actividades emprendedoras a nivel global (Bergmann, Mueller \& Schretlle, 2014). Esta organización compara las actividades emprendedoras de todos los países y determina cuanto influye la actividad emprendedora en el crecimiento económico, además descubre factores que determinan la naturaleza sobre la actividad emprendedora en cada país, promoviendo políticas que desarrollen el emprendimiento.

Si bien la actividad del emprendimiento empresarial tiende a afectar de modo favorable al crecimiento económico, su eficacia podría ayudar aún más, en otra investigación sobre el emprendimiento que incluyó 90 regiones correspondientes a 13 países europeos, encontraron que la calidad influye en el impacto del emprendimiento sobre el crecimiento regional, de modo que dicho aspecto institucional era inclusive más importante que la actividad empresarial privada. La importancia que genera la actividad empresarial para el crecimiento económico influye no solo en los estudios a nivel de países, regiones y sectores económicos, sino también de las ciudades donde existen altos niveles de emprendimiento que fortalecen a la parte económica, ayudando a los individuos que realizan las actividades y a sus calidades de vida.

Para ello el GEM analiza como la sociedad valora la actividad emprendedora y su predisposición hacia ella, es decir las oportunidades que se presentan en el mercado y su capacidad para aprovecharlas y así asegurar el éxito de los negocios. Sin embargo, un entorno que se desarrolla de manera favorable con los emprendedores motiva actitudes positivas hacia el emprendimiento para que los individuos tomen la decisión de iniciar un nuevo negocio y poder aportar con el desarrollo de la economía tanto de ellos como de la sociedad en la que viven. 
McMullen \& Shepherd (2006) mencionan que una vez percibidas las oportunidades, las personas toman la decisión sobre si les conviene y si es viable de aprovecharlas, de igual manera afirman que durante esta etapa, el temor al fracaso puede convertirse en un factor importante para emprender o no hacerlo, por lo tanto el GEM evalúa las percepciones y actitudes individuales que las personas poseen hacia el emprendimiento, cuanto conocimiento tienen y entienden sobre el emprendimiento y como se lo podría poner en marcha.

Existe indicadores que logran hacer relaciones con distintas variables mismos que influyen en el desarrollo del emprendimiento demostrando que las percepciones y actitudes de los emprendedores responderían más a aspectos culturales y políticos propios de una región y no a modelos económicos desarrollados según el nivel que presentan los países.

\section{Metodologia.}

La investigación es de tipo Descriptiva ya que parte de una recopilación bibliográfica de información representativa de los últimos cinco años sobre el emprendimiento y sus indicadores en los países de Ecuador y Perú, el indicador central en el que nos basaremos es la TEA, las fuentes utilizadas fueron informes del "Monitor Global de Emprendimiento" y Global Entrepreneurship Monitor" GEM, artículos académicos y científicos. El análisis del comportamiento del emprendedor desde una perspectiva social y económica, los beneficios que han logrado tener durante los cinco últimos años de Ecuador y Perú, las diferencias entre los emprendedores ecuatorianos y peruanos, la factibilidad que tienen de emprender en cada uno de estos países y lo que representan para el PIB de las naciones estudiadas.

Aún que es de conocimiento de los expertos en este tipo de análisis que hay muchas perspectivas del emprendimiento que, con el análisis de los indicadores actuales, no se puede tener una información completa, si se puede crear una relación entre los emprendimientos de cada país, individualmente y a su vez realizando una comparación a nivel regional y mundial.

Además, es experimental debido al análisis de información estadística de los principales indicadores de emprendimiento de Ecuador y Perú, los cuales han sido relacionados para observar el comportamiento que han tenido individualmente y lo que representan los dos países para la comunidad de América Latina, frente a la comunidad europea.

\section{Resultados.}

\section{El contexto del emprendimiento en América latina.}

Hoy por hoy se considera al emprendimiento como un factor económico, factor que es empleado por diversos individuos como "la economía emprendedora" Audretsch \& Thurik (2001), por esta razón se puede decir que el emprendimiento a nivel mundial se encuentra 
presente en el sector económico, cabe recalcar que ciertos emprendedores se encuentran inspirados por las oportunidades que se les presentan y otros por necesidades, muchos de los países desarrollados han definido un modelo de emprendimiento nuevo fundamentado en la innovación y en la investigación científica, relacionándolo con el progreso de la tecnología Zapata, Fernández \&Neira (2018).

En una investigación realizada por Montoya (2004) se refiere al desarrollo tecnológico y científico como una herramienta esencial para la obtención y cumplimiento del desarrollo económico, la tecnología se puede convertir en una desventaja para los gobiernos que no posean los equipos apropiados para el desarrollo de los procedimientos de innovación tecnológica.

Las características sociodemográficas, económicas y culturales actúan como factores que intervienen directamente en el emprendimiento, particularidades que de acuerdo con las grandes economías son una contribución esencial para que tengan futuro los proyectos Costa, Reyes, \& Soriano (2007), al respecto, un gobierno que no cuente con él la base del autoempleo dentro de sus prioridades corre el riesgo de hallarse en la obstrucción de su desarrollo social y económico Pedroso (2017).

Por lo anterior, es preciso comprender el dinamismo del emprendimiento en el sector económico, en esta región la economía cuenta con un número pequeño de organizaciones innovadoras y que recién han surgido debido a los grandes impedimentos que preexisten y que impiden el surgimiento de estas pequeñas organizaciones Amorós (2011). Referente a esto, Latinoamérica cuenta con una "economía dirigida", es decir, que no tiene ningún tipo de innovación tecnológica, los servicios y productos que ofertan las organizaciones no poseen el valor agregado suficiente Amorós \& Acs (2008), concibiendo de esta forma repetitivos emprendimientos, por lo que en diversos casos decaen por el mercado competitivo existente.

Para Amorós (2011), estos estados no cumplen con el rendimiento esperado dentro del mercado competitivo de esta actividad cuentan, sin embargo, gozan de una cantidad extensa de empresarios, sustentando de este modo que esta área no es generadora de innovadores emprendimientos, originando que tengan poca incidencia sus emprendimientos a nivel integral, apuntando que de estos emprendimientos un $10.8 \%$ poseen una preferencia por temas de internalización, sin embargo, son pocos competitivos estos emprendimientos debido a que no se encuentran vinculados con el progreso tecnológico Amorós, Etchebarne \& Felzensztein (2012).

Gil (2017) menciona que diferentes negocios se localizan en los reglones primarios, afines con las exportaciones de alimentos, siendo transportadas por firmas grandes que trasladan parte de sus ingresos. Por otro lado Sanguinetti (2013) en una publicación determina que el punto clave para el progreso económico es el incremento productivo, haciendo hincapié en 
el desenvolvimiento científico, el cual facilita la generación de métodos de producción, apertura a mercados nuevos, continua mejora en las áreas administrativas, entre otros, unido este fenómeno a la innovación tecnológica y con los diferentes factores productivos hace que se efectúen emprendimientos sustentables y sostenibles en el futuro.

En otras palabras, Latinoamérica a lo largo de su historia y progreso económico ha adquirido un bajo desempeño en la aplicación de los referidos factores de innovación y producción, conllevando a la economía a una dependencia total de los países desarrollados.

Para esclarecer la definición sobre el emprendimiento se ha tenido que instaurar una correlación entre el TOTAL EARLY-STAGE ENTREPRENEURIAL ACTIVITY [TEA], que es el porciento de la población en edades comprendidas entre los 18 y 64 años de edad, que se encuentra en el proceso de creación de un negocio o que ya cuenta con él y que han perdurado por menos de 42 meses en el mercado, todo esto para efectuar una comparación con el porciento de innovación que poseen estos emprendimientos, para tener de este modo una claridad de lo que sucede en Latinoamérica, relacionándolo además con la tasa de cierres de las organizaciones, que es valor porcentual de la población que se halla entre los 18 y 64 años que en el último año ha cerrado un negocio, datos conseguidos del Global Entrepreneurship Monitor (2017).

En esta área de estudio es preciso conocer en la región cómo está la cultura emprendedora, siendo necesario tener un conocimiento de su definición la cual se entiende como el grupo de talentos, valores, normas, conocimientos conformado como una fundamental estrategia, que significativamente interviene en los sistemas económicos y políticos Inglehart (s.f.), simultáneamente es transcendental conocer cuáles son los factores que intervienen sobre el emprendimiento, los cuales pueden favorecer o entorpecer el surgimiento y el perfeccionamiento de esta actividad.

Los factores de acuerdo al Global Entrepreneurship Monitor (2017), que estipulan la cultura emprendedora de un gobierno son: los recursos financieros que son necesarios para la realización de determinada tarea o actividad, este factor unido a las políticas del gobierno deben suministrar las posibilidades y el soporte preciso para la puesta en marcha de los emprendimientos, de igual modo, debe coexistir la indagación y perfeccionamiento de todo lo que facilite representar oportunidades comerciales novedosas, estas deben ser aprovechables para los emprendimientos pequeños y para los que se encuentran en fase de desarrollo, conjuntamente, la creación de mercados interfiere también en la cultura emprendedora, la cual contiene los arreglos comerciales y que no deben tolerar permutaciones sistemáticas, evidenciándose un económico desequilibrio en el estado donde se lleva a cabo esta actividad. 
Aditivo a esto, Kantis (2009) mantiene que la dinámica productiva y la estructura incurren también en la cultura emprendedora, estipulando la práctica profesional y laboral que pueden alcanzar los individuos antes de ingresar al universo del emprendimiento, ayudando así de algún modo al logro de adquisición, desarrollo, práctica y experiencia de nuevos talentos, por lo que poseen una estrecha relación en la realización de los emprendimientos, marcando además que la cultura emprendedora depende tanto de los aspectos individuales como de la estructura productiva, siendo esta la capacidad para poder asumir el liderazgo, la creatividad y los riesgos, mismos que se relacionan directamente con el emprendimiento y, conjuntamente establecen la cultura emprendedora.

\section{Razones que conllevan a emprender.}

Varios motivos son los que existen para el emprendimiento en Latinoamérica, siendo las más fundamentales: el emprendimiento por oportunidad y el emprendimiento por necesidad, de acuerdo al informe del Global Entrepreneurship Monitor (2017), de cada 3 jóvenes, 2 emprendedores de América Latina se encuentran estimulados por la oportunidad y no por la necesidad, donde la oportunidad es una motivación para favorecer el estilo de vida, la independización y el desarrollo económico, por otro lado los individuos que no han podido encontrar un trabajo estable son los emprendedores por necesidad, donde la causa primordial es la falta de empleo, la Organización Internacional del Trabajo (OIT) en el 2017 emitió un informe donde manifestaba que en este año creció el número de desempleados en un 8,4\% más que en el año 2016 que fue de 7,9\%.

Para el año 2018 existió una breve reducción de este indicador, siendo esta falta de empleo motivo para que las personas desarrollen pequeños y microemprendimientos con el propósito de suplir las necesidades básicas fundamentales de las familias (Organización Internacional del Trabajo, 2017).

La Confederación Española de Organizaciones Empresariales (2018) en un informe sostuvo que gran parte de sus entidades en América Latina corresponden a las MIPYMES (90\% del tejido empresarial y el 28\% del Producto Interno Bruto), donde es superior al de otras economías el porciento de emprendimiento, aunque con un número pequeño de empleados al compararla con el de las economías desarrolladas, sobreviviendo estos emprendimientos por más de dos años en un $45 \%$, si se comparan con las economías europeas que son de un $80 \%$, confirmando con lo aludido anteriormente que la escases de empleo influye positivamente en la generación de negocios nuevos, por otro lado en una publicación realizada por el Banco Mundial (2013) hace alusión a lo siguiente: es un síntoma de desequilibrio dañino el hecho de que coexistan diversas entidades pequeñas, excesivas firmas con crecimiento de poco potencial y pocos emprendedores 'transformacionales', que son fundamentales para la creación de nuevas plazas de trabajo y de calidad, incrementando así la producción. 
Por lo tanto, coexistencia de diversas organizaciones instituidas en el territorio, puede ocasionar en el mercado desequilibrios, debido a que estas pequeñas organizaciones poseen un potencial de crecimiento bajo e invención, por lo que ha sido preciso reconocer cuál es el propósito del emprendimiento y que tan fortalecidos son este tipo de negocios para que puedan perseverar en el mercado y si estos interfieren o no en el desarrollo de la economía.

Dentro del marco emprendedor que presenta el GEM para el análisis del emprendimiento a nivel mundial detalla lo siguiente:

\section{TEA y el emprendedor.}

Según el informe del GEM 2017, Ecuador y Perú lideran el ranking de un total de 54 países, primero y segundo lugar respectivamente, en la "Tasa de emprendimientos Nacientes" que va íntimamente relacionado al factor, capacidades percibidas, es decir la actividad nace debido a que estos emprendedores creen tener la capacidad y lo necesario para poder comenzar con su negocio.

De igual manera va ligado a que el miedo al fracaso es mínimo en estas poblaciones comparadas a nivel de la región, Perú es el país con el menor porcentaje con un 30.7\% y Ecuador se ubica dos puestos detrás con un $31.7 \%$, esto se debe también a la población que se dedica a emprender, en estos países la población joven es la que más se arriesga a emprender, ya que no posee muchas responsabilidades y no tienen mucho que perder, tomando en cuenta que muchos de ellos aun cuentan con el apoyo y respaldo de sus padres en caso de fracaso.

En los últimos años se ha comenzado a hablar de una nueva generación en lo que se refiere al emprendimiento, es denominada la "Generación del milenio", son aquellas personas que han nacido entre 1980 y 2000, en donde los indicadores de temor al fracaso y percepciones son iguales a los demás grupos de emprendedores, pero aquí si sobresale la participación de la mujer.

Adicionalmente de acuerdo al espíritu del emprendedor se toma en cuenta tres características especiales que lo permiten destacar del resto las cuales son la conciencia emprendedora, percepción de las oportunidades y la eficacia personal para emprender.

Perú sobresale con una población con espíritu emprendedor con un valor mayor a 0.37 a diferencia de Ecuador que, aunque no existe una brecha muy grande, se ubica por debajo de Perú con un 0.28.

Haciendo referencia a la intención de emprender que tienen las poblaciones de Perú y Ecuador, más de la mitad de la población ecuatoriana tiene la intención de emprender, pero no se cumple debido a que se contrarresta con el temor al fracaso que existe, la gente no está 
dispuesta a perderlo todo, convirtiendo a esta característica en un obstáculo para continuar con la idea de emprendimiento.

Debemos de tomar en cuenta que la intención comienza con un porcentaje alto al inicio del negocio naciente, pero alrededor de los próximos tres años va disminuyendo constantemente, muchas veces debido a que las expectativas de rentabilidad o utilidad que se generan en estas poblaciones no se ven evidenciada en el desarrollo de su emprendimiento, por lo que existe un gran porcentaje de cierre de emprendimiento en dichos países.

De manera general se presenta que entre los emprendedores pertenecientes a la TEA, alrededor de un $52.1 \%$ son de género femenino, y un $47.9 \%$ pertenecen al género masculino, en estos países, Perú y Ecuador, la participación de la mujer se ve reflejada en dichos índices, gracias a los esfuerzos por tener mayor atención y la falta de oportunidades existentes en el mercado laboral, las mujeres toman la decisión de emprender su propio negocio, y según las estadísticas reflejan que más de la mitad de los emprendimientos realizados por mujeres son las que tienen mayor continuidad, muchos dicen que se debe a la capacidad de ser polifuncionales, tienen una mejor organización ya que no solo se dedican a su trabajo, gran parte de ellas son amas de casa, madres, mujeres que tienen que sacar adelante sus familias.

A nivel regional el porcentaje de participación de las mujeres en emprendedores nacientes es del $52.3 \%$ pero si nos basamos en emprendedores nuevos los hombres son los que se llevan el mayor porcentaje con un $60.2 \%$ de participación.

Un dato relevante es mencionar que el $60 \%$ de la población que emprende está situada en el área urbana, esto se debe a que la gente del campo tiende a tener más dificultad de salir de su zona de confort de la actividad que realiza, muchas veces debido a que carecen de información y no sienten respaldo de las entidades estatales para poder emprender. En Ecuador tiende a tener una leve diferencia en el porcentaje de TEA de la zona urbana, debido a que se motiva a la creación de asociaciones que les permitan tener mayores oportunidades de crecimiento, además el gobierno da capacitaciones en el tema de valor agregado y nuevas formas de negocios.

Sobre la característica del emprendedor respecto al ingreso mensual percibido por hogar, destaca los emprendedores que se encuentran en un rango de $\$ 376$ a $\$ 750$, lo que representa que las poblaciones de la TEA que emprenden son aquellas que se ven con la necesidad de adquirir nuevas fuentes de ingreso, o aquellas que se encuentran en puestos de trabajo en donde no cumplen con sus expectativas tanto laborales como económicas y deciden independizarse.

Tabla 1. Motivaciones de los emprendedores

Oportunidad de mejora (\% en TEA) 


\begin{tabular}{lccccccc}
\hline & TEA & $\begin{array}{c}\text { TEA } \\
\text { oportunidad } \\
(\% \text { en TEA })\end{array}$ & $\begin{array}{c}\text { TEA } \\
\text { necesidad } \\
(\% \text { en } \\
\text { TEA) }\end{array}$ & Ingresos & Independencia & Total & $\begin{array}{c}\text { Índice } \\
\text { motivacional }\end{array}$ \\
\hline Perú & $26,60 \%$ & $80,26 \%$ & $16,66 \%$ & $36,79 \%$ & $27,47 \%$ & $62,26 \%$ & 3,7 \\
Ecuador & $29,62 \%$ & $57,31 \%$ & $42,33 \%$ & $17,87 \%$ & $18,97 \%$ & $36,72 \%$ & 0,9 \\
\hline
\end{tabular}

Fuente: “Global Entrepreneurship Monitor” Ecuador 2017, ESPOL, 2018,

Analizando las motivaciones de los emprendedores por oportunidad de mejora de cada país, Perú destaca en que su mejor motivación es los ingresos, mejorar su nivel de vida, brindar mayores beneficios a sus familias o a ellos mismos, en cambio la motivación para los emprendedores ecuatorianos es la independencia, y a esto nos referimos una independencia económica que a su vez derive en independizarse de las familias, que es el caso de la mayoría de jóvenes, y por otro lado independizarse económicamente del lugar en donde se encuentran laborando.

Figura 1. Países de Latinoamérica con la TEA más alta 2017

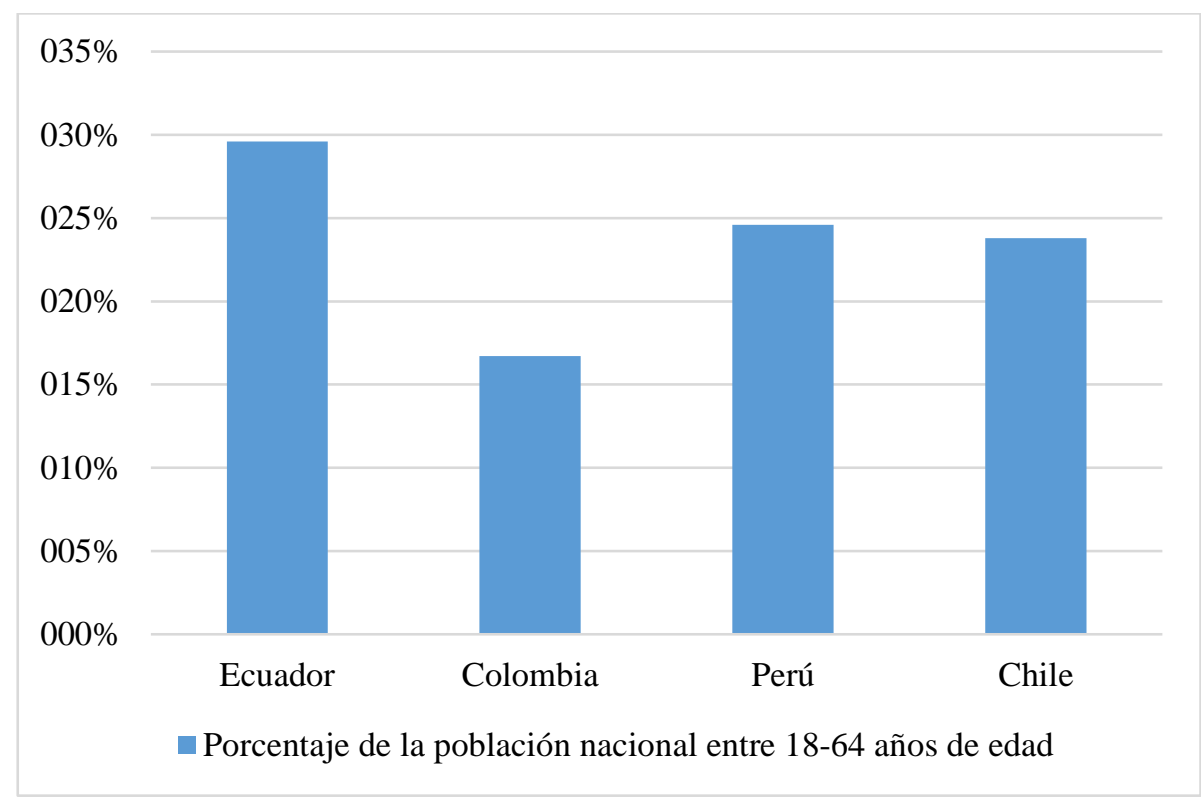

Fuente: "Global Entrepreneurship Monitor" Ecuador 2017, ESPOL, 2018,

En el ámbito social, el factor que predomina en la cultura de estos países líderes respecto al emprendimiento es "El emprendimiento capta mucha atención de los medios" en 2017 debido a que el emprendimiento se ha convertido en un boom para las poblaciones de estos países sin embargo los beneficios de dicha atención mediática no son los suficientes para impulsar 
estos negocios. Con referencia a los otros factores dentro del ámbito social, los países se encuentran en la media a comparación de los países de la región.

\section{Inversión requerida para iniciar un negocio.}

Carriel (2017) menciona que el monto requerido para iniciar un negocio es diferente para cada país, por lo cual se ha optado por usar la mediana a fin de hacer comparaciones, así por ejemplo el promedio en Europa $(\$ 17,200)$, Asia y Oceanía $(\$ 15,200)$, y América del Norte $(\$ 18,500)$ supera al global, esto se debe a que en estas regiones su economía se basa primordialmente en la innovación.

La realidad para los países de Latinoamérica y el Caribe no es distinta, puesto que el monto para iniciar un negocio también varía, el mismo que va desde \$257 en Uruguay hasta \$6,400 en Colombia, siendo los puntos extremos. Para el caso de Ecuador y Perú se requiere un monto de $\$ 2,000$ y $\$ 1,577$ para iniciar un negocio, respectivamente.

Figura 2. Inversión requerida para iniciar un negocio.

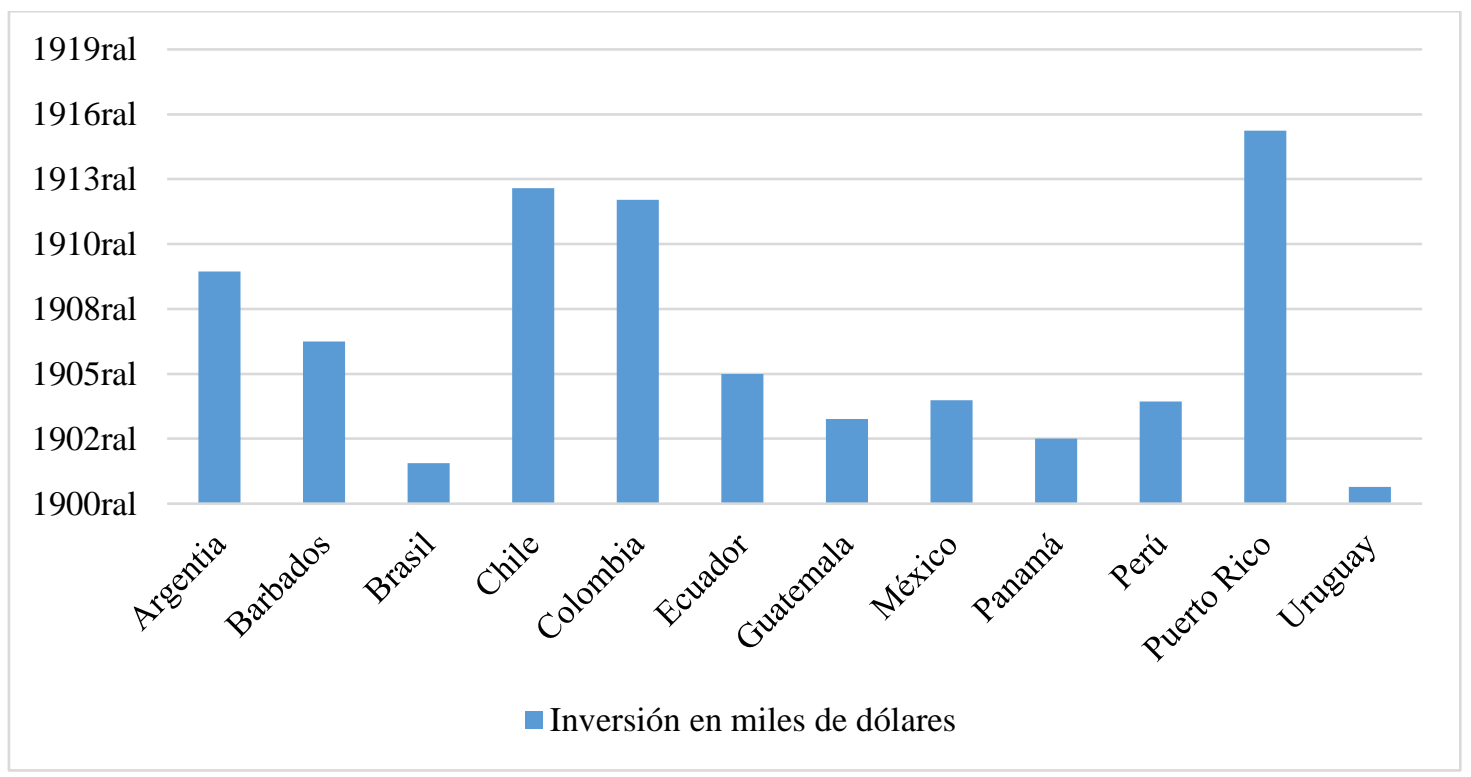

Fuente: “Global Entrepreneurship Monitor” Ecuador 2016, ESPOL 2017

Cabe mencionar que al parecer no existe una correlación entre la cantidad para iniciar un negocio y la TEA de estos países; es decir, que el nivel de emprendimiento en la región dice muy poco (o talvez nada) sobre el tamaño de dichas actividades.

\section{Fuentes de financiamiento.}

Conseguir el financiamiento suficiente se ha convertido en un desafío, ya que es un recurso necesario para todas las empresas, especialmente para aquellas que son de reciente creación 
y se encuentran en crecimiento. La falta de acceso a los recursos financieros o la disposición inoportuna de ellos dificultan los esfuerzos de emprendimiento, logrando que se reduzca el potencial de inversión y crecimiento de las empresas Carriel (2017).

En la región, el 96\% de los actores que se encuentran inmersos en la actividad emprendedora temprana (TEA) menciona utilizar fondos propios ya sea de manera parcial o total para financiar su actividad. En la región el 75\% de la inversión es cubierta por recursos propios; cómo se puede observar en la figura 3, los emprendedores de Ecuador cubren el 74\% de la inversión requerida con fondos propios mientras que en Perú la inversión es cubierta con el $72 \%$ de fondos propios.

Figura 3. Fuentes de financiamiento

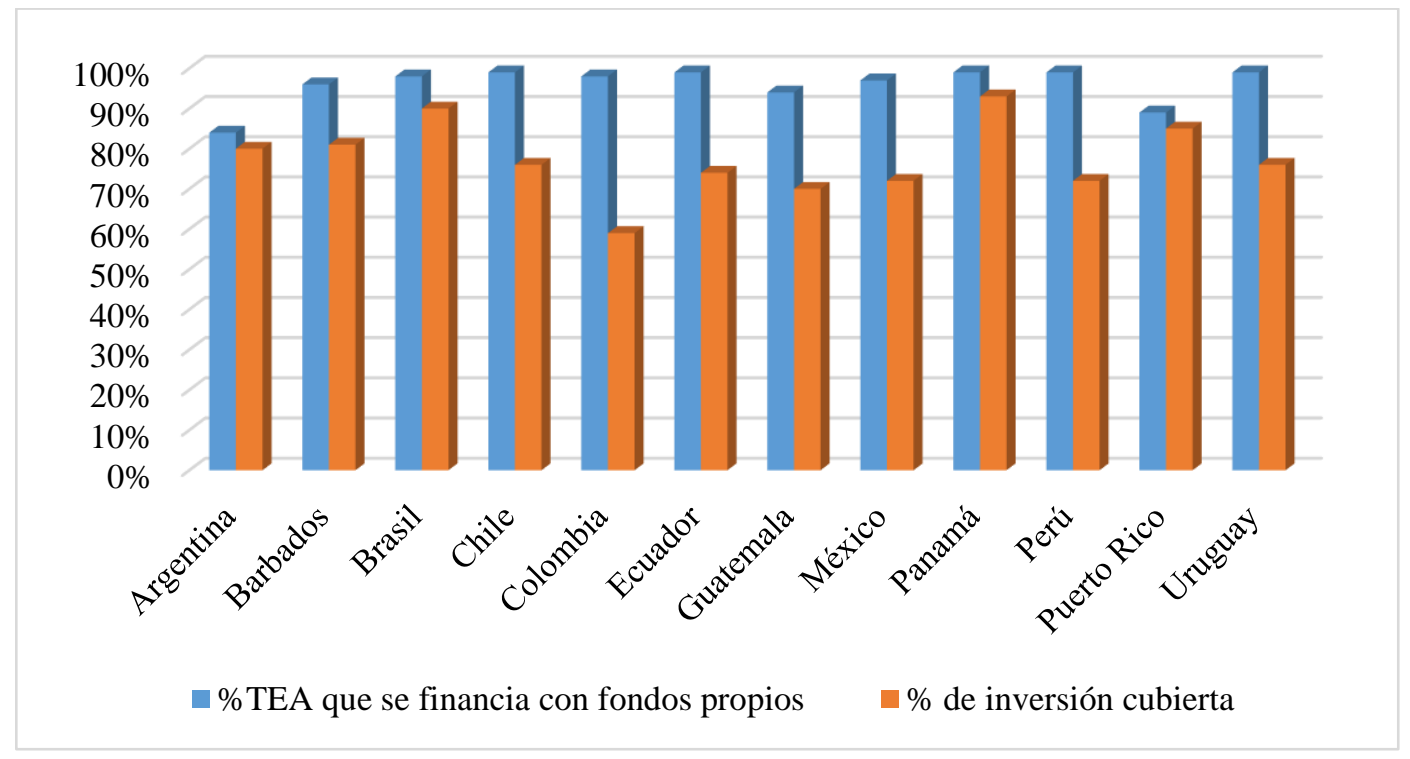

Fuente: “Global Entrepreneurship Monitor” Ecuador 2016, ESPOL 2017

Los expertos de la región han llegado a un consenso en el que mencionan que los emprendedores por lo general carecen de suficientes fondos propios con el fin de financiar sus negocios, como resultado la mayoría financia sus empresas mediante la ayuda de familiares y amigos, u otros inversionistas informales que no se asocian o toman alguna participación.

Es así que el uso de otras fuentes de financiamiento se ha vuelto un tema relevante, sirviendo en la actualidad como indicador de la sostenibilidad de los emprendimientos. Para el caso de la región, las tres fuentes más importantes de financiamiento externo son: familiares, bancos y gobierno. 
Figura 4. Principales fuentes de financiamiento para emprendedores (TEA) en América Latina y El Caribe

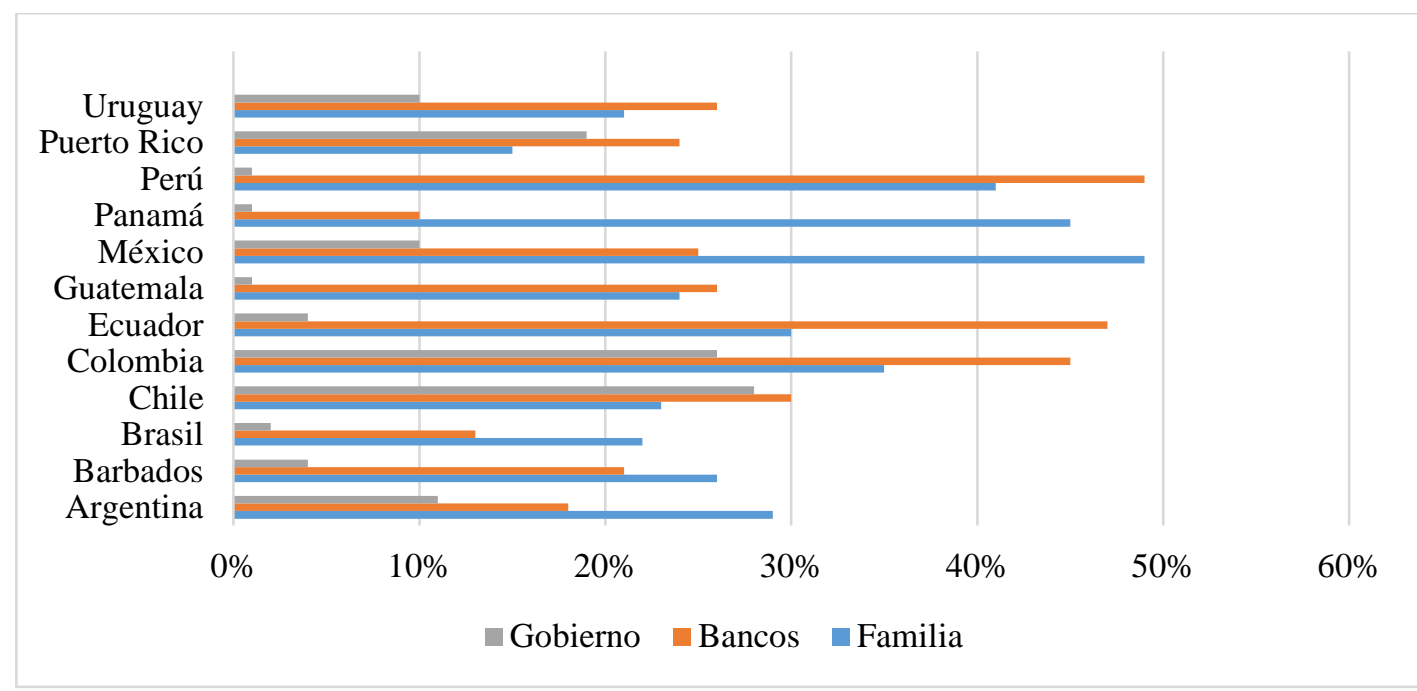

Fuente: “Global Entrepreneurship Monitor” Ecuador 2016, ESPOL 2017

En la Figura 4, se muestra una comparación, donde también se evidencia que la relevancia de cada una de esas fuentes de financiamiento varía de acuerdo al país.

Para el ejemplo, el 30\% de los emprendedores de Ecuador utilizan el financiamiento de familiares, pero en Perú el $41 \%$ de los emprendedores ve a sus familiares como fuente de financiamiento.

De igual manera, existe cierta similitud entre Ecuador y Perú al acudir al financiamiento de una institución financiera, es decir de un Banco, siendo del 47\% y $49 \%$ respectivamente.

Como es notorio la participación del Gobierno en el financiamiento de los negocios de los emprendedores es sumamente baja, siendo para Ecuador su participación del $4 \%$ y para el Perú del 1\%. En la región el porcentaje de financiamiento por parte del gobierno es en promedio del $9,75 \%$.

\section{Giro del negocio Evaluación del entorno.}

El GEM reúne a los sectores productivos de una economía basándose en la Clasificación Internacional Industrial Uniforme (CIIU), en cuatro categorías.

En la categoría de Sectores orientados al consumidor, encontramos negocios como tiendas minoristas, restaurantes y bares, hospedajes, instituciones educativas, servicios de recreación, entre otros. 
Como se puede evidenciar en la figura 5 en este sector es al que dedican mayormente sus actividades emprendedoras tanto Ecuador como Perú.

En Sectores de servicios empresariales donde se encuentran negocios como servicios financieros, servicios de publicidad, construcción, aseguradoras, empresas de outsourcing, entre otros.

Para el caso de Ecuador el porcentaje de actividades emprendedoras que se dedican a este sector es notoriamente inferior en comparación a Perú.

En Sectores de transformación, donde se encuentran negocios relacionados a actividades de transformación de materias primas, transporte de bienes y personas, manufactura y distribución mayorista. En esta categoría Perú es el que mayormente participa en comparación a Ecuador, tanto en sus negocios nacientes, negocios nuevos y los ya establecidos.

Para la última categoría tenemos a los Sectores extractivos cuyas actividades se orientan a la extracción de recursos naturales, como la agricultura, la ganadería, la pesca y la minería.

En este caso Ecuador y Perú coinciden en el porcentaje de participación de sus actividades emprendedoras en negocios ya establecidos siendo éste del 16\%, sin embargo, es Ecuador el que mayormente aporta a este sector a través de sus negocios nacientes y nuevos, cuyo porcentaje es del $10 \%$ y $12 \%$ respectivamente.

Figura 5. Distribución de actividades por tipo de industria

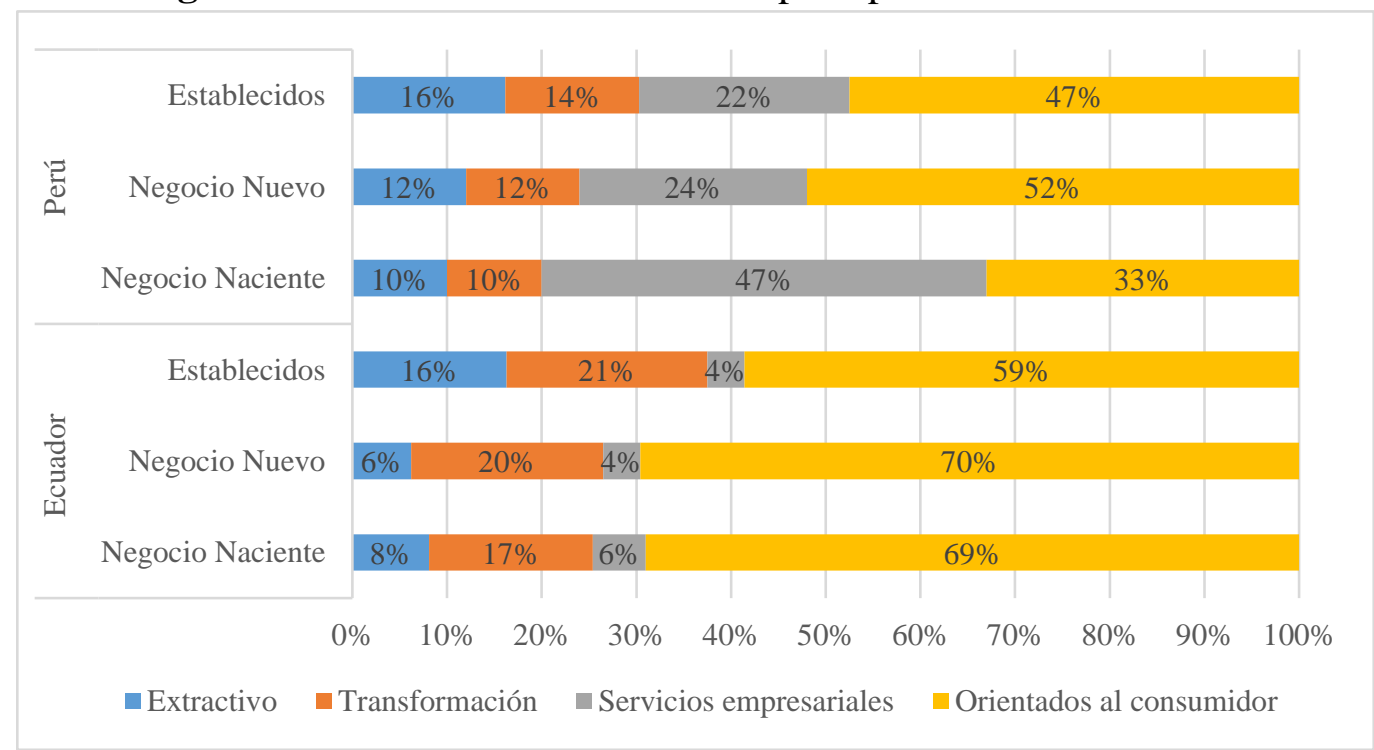

Fuente: “Global Entrepreneurship Monitor” Ecuador 2017, ESPOL 2018 


\section{La actividad Intra- Emprendedora.}

De acuerdo al Global Entrepreneurship Monitor (2017), la Actividad Emprendedora del Empleado (EEA, por sus siglas en inglés) muestra el porcentaje de empleados que se encuentran involucrados en alguna actividad relacionada a un intra-emprendimiento en una empresa. Estas actividades pueden tratarse de la creación de un nuevo producto o servicio para el mercado, ya sea a través de la creación nuevas líneas de negocio, liderando proyectos sobre mejoras de procesos, o a su vez mediante la invención de una nueva área administrativa, etc.

Un alto nivel de EEA va relacionado por lo general con el nivel de innovación que tienen las empresas y su apertura para que sus empleados participen en actividades que den origen a nuevas oportunidades de negocio o fortalezcan el desempeño de las mismas.

Figura 6. Actividad Emprendedora del Empleado (EEA)

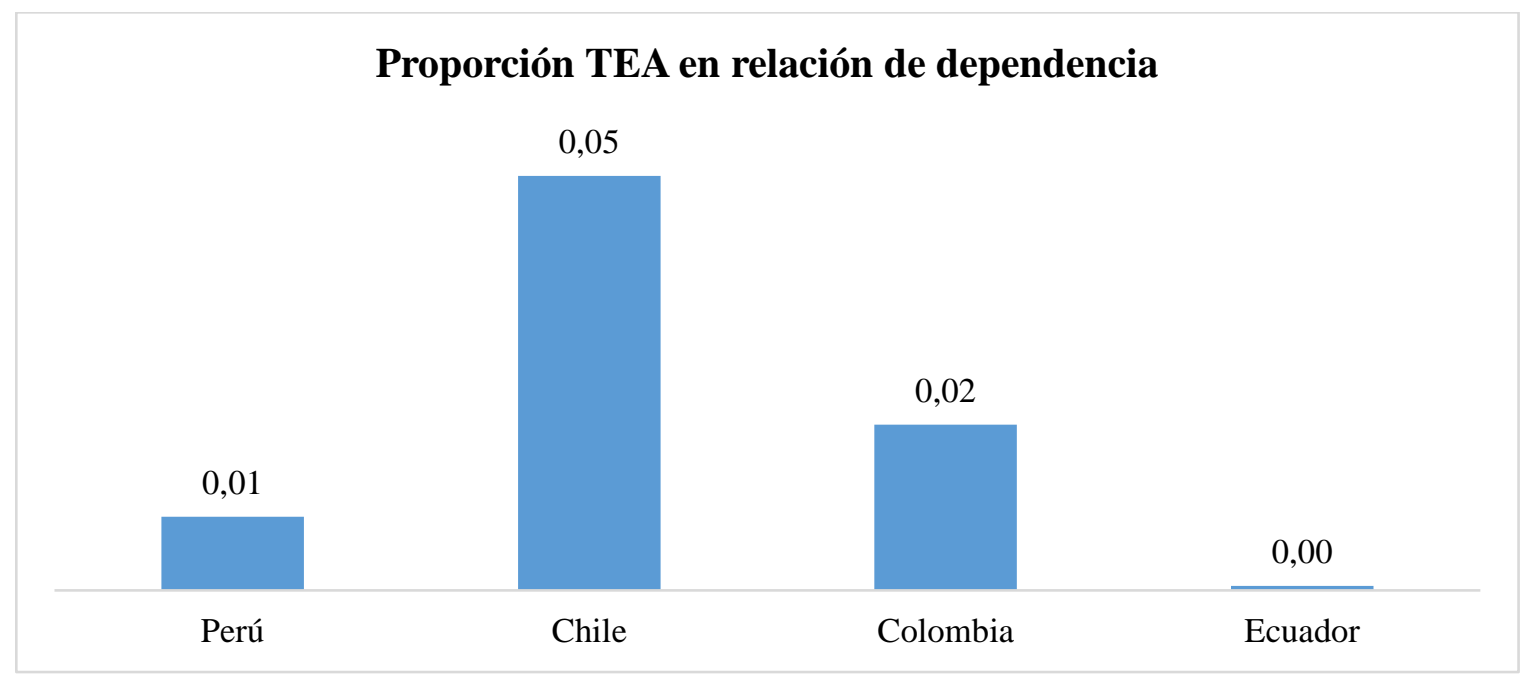

Fuente: “Global Entrepreneurship Monitor” Ecuador 2016, ESPOL 2017

Para el 2017, Ecuador mantiene la EEA más baja de la región, siendo ésta del $0.5 \%$ de la población adulta empleada, el porcentaje para Perú no difiere mucho, siendo del $0.8 \%$, lo que demuestra que en ambos países aún no se han tomado las acciones necesarias para que la cultura empresarial promueva la colaboración y empoderamiento de los empleados en proyectos de innovación. Esta circunstancia puede deberse también a que el ecosistema emprendedor de Ecuador y Perú no se encuentran al nivel de desarrollo de países como Chile, país que lidera la lista de intra-emprendimiento en la región con un porcentaje del $4.46 \%$. Bien Chile se podría mencionar como un caso particular ya que su nivel de EEA se encuentra muy por encima del promedio de la región el cual es del 1.58\%, convirtiéndose en un 
referente regional en la edificación de un ecosistema que fomenta la innovación tanto dentro y fuera de las empresas.

La Tabla 2 reúne ejemplos de países con TEAs y EEAs similares. Como se puede evidenciar, Ecuador se encuentra en el cuadrante de una TEA alta y una EEA baja junto con Perú y Guatemala. Por otro lado, los países que evidencian una TEA baja y una EEA alta son países desarrollados como Reino Unido y Luxemburgo, lo que demuestra que el nivel de desarrollo de un país no se encuentra necesariamente relacionado a una TEA alta.

Tabla 2. Comparación países TEA y EEA

EEA alta $\quad$ EEA baja

TEA Alta Estonia, Canadá $\quad$ Ecuador, Perú y Guatemala

TEA Baja Reino Unido, Luxemburgo Bulgaria, Bosnia y Herzegovina Grecia

Fuente: “Global Entrepreneurship Monitor” Ecuador 2017, ESPOL 2018

\section{Evaluación del entorno.}

Tabla 2. Evaluación del entorno de emprendimiento

\begin{tabular}{lll}
\hline \multicolumn{1}{c}{ Factor } & \multicolumn{1}{c}{ Ecuador } & Perú \\
\hline Apoyo financiero & 3,15 & 3,59 \\
Educación y Formación. & 5,99 & 5,19 \\
Infraestructura comercial y profesional. & 5,06 & 4,31 \\
Apertura mercado interior. & 4,59 & 4,22 \\
Acceso a Infraestructura física. & 6,99 & 5,84 \\
Políticas Gubernamentales & 3,58 & 4,57 \\
Normas Sociales y Culturales & 4,99 & 5,47 \\
\hline
\end{tabular}

Fuente: “Global Entrepreneurship Monitor” Ecuador 2017, ESPOL, 2018.

Apoyo Financiero: Se puede observar que en los estándares financieros entre Perú y Ecuador existe una clara diferencia, es así que Ecuador consta con $3.15 \%$ de inversión, mientras que Perú tiene un valor de $3.59 \%$, es decir que existe una diferencia del $0.44 \% \%$ de inversión.

Lo que lleva a concluir que en Ecuador el apoyo financiero tiene un déficit, debido a que Perú dentro de sus valores financieros, consta con mayores ingresos que se encuentran representados en el PIB. 
Políticas Gubernamentales: En las políticas gubernamentales, que benefician al emprendedor, se percibe claramente que en Perú existen más garantías para que se pueda desarrollar un emprendimiento sustentable. La diferencia radica en sus porcentajes, Ecuador con $3.53 \%$ y Perú con $4.40 \%$, la diferencia es muy notable, lo que ayuda a entender que Ecuador no consta con las garantías necesarias para un emprendimiento sustentable.

Políticas Gubernamentales Regulación: En cuanto a las políticas gubernamentales de regulación, existe una diferencia del $0.54 \%$, debido a que Ecuador consta con $2.93 \%$ y Perú con 3.47, por lo tanto, esto quiere decir que en Ecuador es mucho más difícil obtener los permisos necesarios para poder emprender, debido a los reglamentos internos que se han ido manejando a lo largo de los años.

Educación y Formación: En esta categoría Ecuador y Perú constan con una diferencia del $0.05 \%$, es decir, que la diferencia no es mucha por lo que se encuentran a la par.

En los últimos 10 años estos países han mejorado su educación secundaria lo que ha hecho que en exista un mayor seguimiento en la educación de tercer nivel, con lo que han logrado una educación continua en estas áreas.

Las ideas innovadoras son las que impulsan el emprendimiento lo que ha hecho que estos países que encuentren ocupando los lugares más altos en la TEA.

Transferencia de I+D: Dentro de esta categoría los dos países se encuentran casi empatados, con tan solo $0.02 \%$ de diferencia, es decir que las dos naciones tienen casi la misma capacidad tecnológica y de innovación para sus proyectos de emprendimiento.

Hay que resaltar que estos valores son solo a nivel regional, puesto que si se los pone a comparar con países europeos las estadísticas variarían drásticamente, ya que Europa es líder en innovación por sus capacidades tecnológicas.

Acceso a Infraestructura física: En este punto en particular Ecuador se encuentra por encima de Perú con una diferencia de $1.15 \%$, es así como en Ecuador el acceso y costo a diferentes equipos para emprendimiento es más accesible y de la misma forma la vialidad para obtener recursos servicios básicos y TICS.

Normas Sociales y Culturales:

En esta categoría Ecuador se encuentra por debajo de Perú, puesto que Perú cuenta con un valor de 5.47 y Ecuador 4.99, es decir que en este país no se otorga la seguridad y autonomía al emprendedor, por esta razón se ha tratado de implementar nuevas políticas para que no exista un retroceso al momento de que una persona quiera empezar un negocio. 
Se espera que estas nuevas políticas se apliquen en este año.

\section{Internacionalización de productos o servicios}

Figura 8. Proporción de ingresos que provienen de clientes extranjeros, negocios en TEA

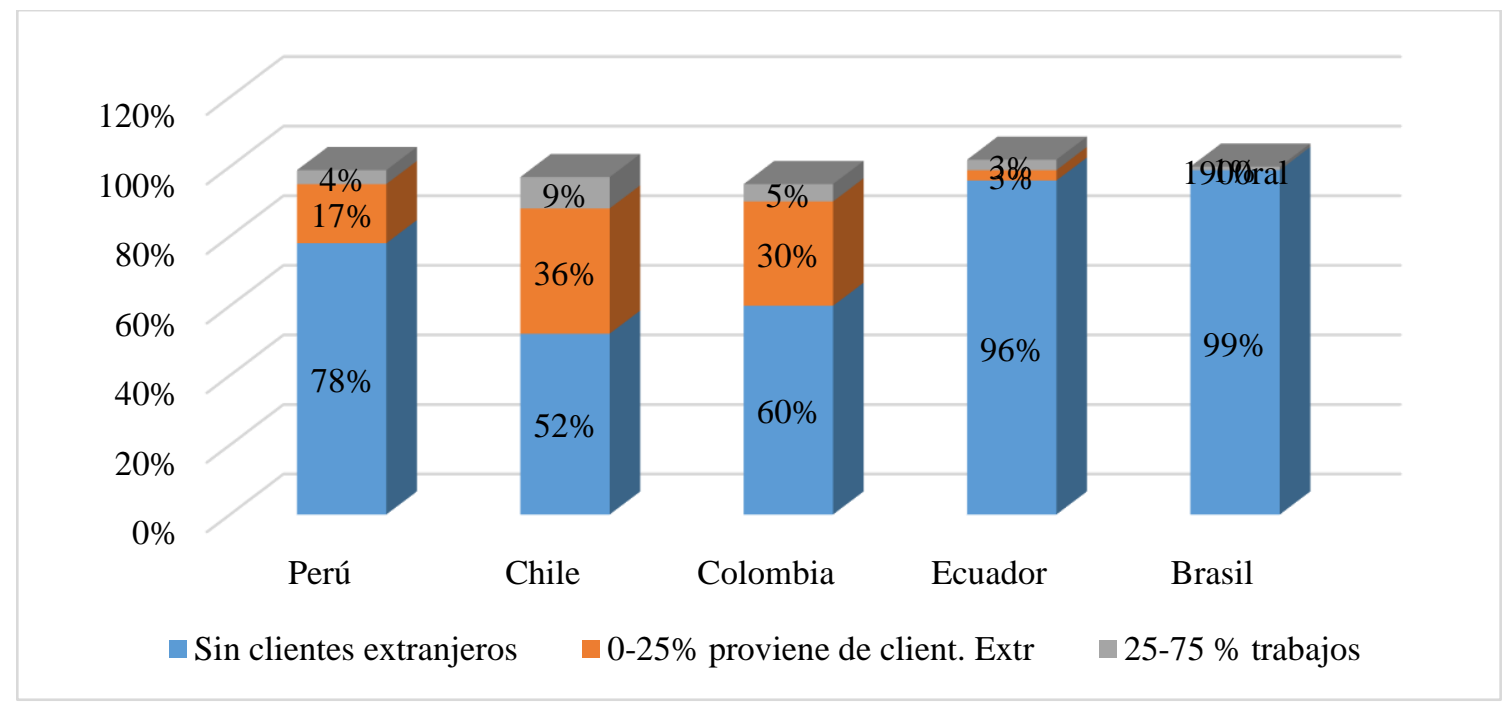

Fuente: “Global Entrepreneurship Monitor” Ecuador 2017, ESPOL 2018

En el año 2017, el 95.6\% de los negocios ecuatorianos en la TEA, tuvieron exclusivamente clientes nacionales, ubicando de esta forma a Ecuador como el segundo país con mínimo nivel de internacionalización de la región en sus negocios luego de Brasil con un 99.3\%, en tercer lugar, se ubica Perú con el 78.07\%. Por el contrario, países como Colombia y Chile evidenciaron un mayor nivel de internacionalización. En este sentido se evidencia que Ecuador y Perú no han sido eficientes en destinar mayores esfuerzos en el comercio internacional, careciendo de un proceso de concienciación y sobre todo de estrategias a largo plazo que ayuden a su producto o servicio a ser mejor al de su competencia de tal forma que pueda internacionalizarse.

\section{Conclusiones.}

- El GEM desde su creación ha tenido el importante papel de brindar información verdadera y comparable que permita realizar una comparación compleja entre el emprendimiento y el crecimiento económico a través de encuestas que ayudan a identificar los factores que fomentan o limitan la actividad emprendedora. De esta forma el GEM clasifica a los países de acuerdo con la fase de desarrollo económico en que se encuentran sustentado en el PBI per cápita y la proporción de bienes primarios en las exportaciones, entre otros indicadores. Estas categorías son: 
economías basadas en recursos, economías basadas en eficiencia y economías basadas en innovación. Para el caso de Ecuador y Perú, estos países se ubican en una economía basada en eficiencia si bien es cierto ya no es una economía basada en recursos, pero tampoco es una economía basada en innovación que sería el punto óptimo.

- Dentro de las principales razones que motivan a emprender en Latinoamérica se encuentra: el emprendimiento por oportunidad mismo que alude a favorecer el estilo de vida, la independización y el desarrollo económico; y el emprendimiento por necesidad, opción elegida debido a que las personas no lograr obtener un trabajo estable. Conforme al porcentaje sobre las motivaciones de los emprendedores por oportunidad de mejora, Perú destaca en que su mejor motivación son los ingresos con un $36,79 \%$, mejorar tanto su nivel de vida como el de su familia, en cambio la motivación para los emprendedores ecuatorianos es la independencia con un 18,97\%, lo que demuestra que buscan independizarse económicamente del lugar en donde se encuentran laborando como de su familia.

- De acuerdo el informe del GEM 2017, Ecuador y Perú lideran el ranking de un total de 54 países, primero y segundo lugar respectivamente, siendo los países más emprendedores de la región con una Tasa de emprendimientos Nacientes (TEA) para Ecuador que alcanza el 29,62\% y para Perú el 26,60\%. La participación de la mujer se ve reflejada en estos índices de manera que entre los emprendedores pertenecientes a la TEA, alrededor de un $52.1 \%$ son de género femenino, y un $47.9 \%$ corresponde al género masculino, en ambos países, esto como resultado de la falta de oportunidades existentes en el mercado laboral, por lo que las mujeres toman la decisión de emprender su propio negocio, y según las estadísticas reflejan que más de la mitad de los emprendimientos realizados por mujeres son las que tienen mayor continuidad, lo que puede deberse a la capacidad de la mujer de ser poli funcional.

- Sin duda alguna el financiamiento se ha vuelto una herramienta fundamental para los emprendimientos nacientes y en crecimiento, ya que es un medio para lograr sus objetivos y poder consolidarse en el mercado, en aras de un día poder internacionalizar su producto o servicio. A pesar de que los emprendedores de Ecuador cubren el $74 \%$ de la inversión requerida con fondos propios mientras que en Perú la inversión es cubierta con el $72 \%$ de fondos propios, ambos países buscan financiar sus negocios a través de sus principales fuentes, siendo éstos: familiares, bancos y gobierno. En Ecuador y Perú se evidencia que a la fuente de financiamiento que más recurren es a la de una institución financiera, es decir de un Banco, siendo del $47 \%$ y $49 \%$, seguido de los familiares con $30 \%$ y $41 \%$, respectivamente. Por otro lado el apoyo del gobierno en el financiamiento de los emprendedores es muy débil, en Ecuador el aporte del gobierno es del $4 \%$ y en Perú es del $1 \%$ por lo que los gobiernos de éstos países tiene el papel fundamental de establecer mecanismos que 
faciliten el acceso de los emprendedores al financiamiento, a través de bancos o agencias gubernamentales donde los criterios de evaluación para acceder al préstamo no sean basados en activos sino tanto en la calidad de la idea como la calidad del emprendedor; además de promover la creación de fondos de capital semilla.

- Como se ha establecido anteriormente, Latinoamérica una "dirigida economía", es decir, que no posee ningún tipo de innovación tecnológica, por lo que los servicios y productos carecen de valor agregado suficiente, esto debido a características sociodemográficas, económicas y culturales, factores que intervienen en el emprendimiento, concibiendo de esta manera repetitivos emprendimientos que decaen por el mercado competitivo existente. Esto se puede reflejar en la Actividad Emprendedora del Empleado (EEA) la cual muestra el porcentaje de empleados que se encuentran involucrados en alguna actividad relacionada a un intraemprendimiento en una empresa. En este ámbito Ecuador mantiene la EEA más baja de la región, siendo ésta del $0.5 \%$ de la población adulta empleada, el porcentaje para Perú no difiere mucho, siendo del $0.8 \%$, lo que demuestra que los emprendedores son fácilmente superados por competidores internacionales que cuentan con tecnologías más avanzadas. De aquí que recae la importancia que ambos países implementen una cultura empresarial que permita ser y convierta a sus empleados en los actores principales en los proyectos de innovación que desarrolle la empresa, de tal forma que sean ellos quienes construyan un ecosistema que fomente la innovación tanto dentro como fuera de ésta.

- Una vez realizado una evaluación del entorno en ambos países se demuestra que en el Factor de Políticas Gubernamentales Perú es el país donde existen más garantías para que se pueda desarrollar un emprendimiento sustentable donde su porcentaje alcanza el $4.40 \%$ y el de Ecuador el 3.53\%. En el factor de Educación y Formación Ecuador y Perú van casi a la par con un 5,99\% y un 5,19\% respectivamente, dado que en los últimos 10 años estos países han mejorado su educación secundaria lo que ha logrado que exista un mayor seguimiento en la educación de tercer nivel. Por otro lado en el factor Acceso a Infraestructura física Ecuador se encuentra por encima de Perú con una diferencia de $1.15 \%$, lo que indica que en Ecuador el acceso y costo a diferentes equipos para emprendimiento es más accesible y de la misma forma la vialidad para obtener recursos servicios básicos y TICS.

\section{Referencias bibliográficas.}

Amorós, J. (2011). El proyecto Global Entrepreneurship Monitor (GEM): una aproximación desde el contexto latinoamericano. Academia. Revista Latinoamericana de Administración, 1-15.

Andrade Espinoza, X. (2012). Las trabas al emprendimiento en Ecuador. RETOS, 2, 2-12. 
Bernabé Argandoña, L., Hidalgo Arriaga, J., \& Rodas Hidalgo, F. (2016). Indicadores del Emprendimiento. Análisis de 12 países latinoamericanos. Revista Publicando, 1-10.

Carriel Eliot, E. (2017). El Emprendimiento en Ecuador: Visión y Perspectivas. 109.

Confederación Española de Organizaciones Empresariales. (2018). Las mipymes generan el $28 \%$ del PIB en Latinoamérica pero carecen aún del impulso necesario. Obtenido de https://www.ceoe.es/es/contenido/actualidad/noticias/las-mipymes-generan-el-28del-pib-en-latinoamerica-pero-carecen-aun-del-impulso-necesario

De la Torre, A. (2013). Banco Mundial: Detrás de la escasez de empresarios innovadores en América Latina. Obtenido de https://www.bancomundial.org/es/news/pressrelease/2013/12/05/world-bank-latin-america-shortage-innovative-entrepreneurs

Gestión. (2018). Perú vs. Ecuador: Cuatro datos claves del partido en la cancha del emprendimiento. Obtenido de https://gestion.pe/economia/managementempleo/peru-vs-ecuador-cuatro-datos-claves-partido-cancha-emprendimiento250092-noticia/?outputType=amp

Gil Gaytán, O. (2017). Association between the profile of the entrepreneur and success in international entrepreneurship. Revista de Tecnología y Sociedad.

Global Entrepreneurship Monitor. (s.f.). ¿Qué es GEM? Obtenido de https://gem.ufm.edu/que-es-gem/

Inglehart, R. (s.f.). Modernización y posmodernización. Recuperado el 2 de enero de 2020, de https://dialnet.unirioja.es/servlet/libro?codigo=175334

Kantis, H., \& Drucaroff, S. (2009). Emprendimiento corporativo en América Latina. (N. Otsuka, Ed.)

Landsdale, D., Abad, C., \& Vera, D. (s.f.). Impulsores Claves para Establecer el Ecosistema Dinámico de Emprendimiento en Ecuador. 1-7.

Lasio, V., Ordeñana, X., Caicedo, G., Samaniego, A., \& Izquierdo, E. (2018). Global Entrepreneurship Monitor: Ecuador 2017. (ESPOL, Ed.) 10-80.

Lecaro, E., Trujillo , E., Villacís, K., Coello , R., \& Palacios, R. (2017). Global Entrepreneurship Monitor: Ecuador 2016. (ESPOL, Ed.) 10-82.

León-Mendoza, J. (2019). Emprendimiento empresarial y crecimiento económico en Perú. Estudios Gerenciales, 429-439. 
Pedroso, J. (Marzo de 2017). El fenómeno emprendedor. Obtenido de https://www.fraternidad.com/descargas/emprendedor/Fenomeno\%20Emprendedor \%20Fraternidad-Muprespa.pdf

Revista Líderes. (2015). Ecuador y Perú entre las economías más emprendedoras del mundo, según reporte. Obtenido de https://www.revistalideres.ec/lideres/ecuador-perueconomias-emprendedoras.html

Sanguinetti, P. (2013). Emprendimientos en América Latina: Desde la subsistencia hacia la transformación productiva. (CAF, Editor) Obtenido de https://www.microfinancegateway.org/sites/default/files/mfg-es-documentoemprendimientos-en-america-latina-desde-la-subsistencia-hacia-la-transformacionproductiva-2013.pdf

Serida, J., Guerrero, C., Alzamora, J., Borda, A., \& Morales, O. (2017). Global Entrepreneurship Monitor: Perú 2016-2017. 12-70.

Guerrero, C., Alzamora, J., Borda, A., \& Morales, O. (2018). Global Entrepreneurship Monitor: Perú 2017-2018. 11-72.

Valencia Maldonado, G., \& Erazo, M. A. (2017). Indicadores de Emprendimiento. Caso Ecuador. Revista Publicando, 1-8.

Zamora Boza, C. (2018). La importancia del emprendimiento en la economía: el caso de Ecuador. Espacios, 15.

Zapata, G., Fernández López, S., \& Neira Gómez, I. (2018). El emprendimiento tecnológico en Suramérica: una aproximación a sus determinantes individuales. Perfiles latinoamericanos, 26.

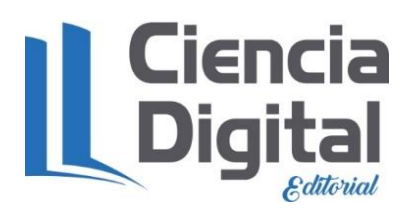




\section{PARA CITAR EL ARTÍCULO INDEXADO.}

Villalba Díaz, M. F. (2020). La cultura del emprendimiento de Ecuador en relación a Perú. Visionario Digital, 4(3), 147-169. https://doi.org/10.33262/visionariodigital.v4i3.1340

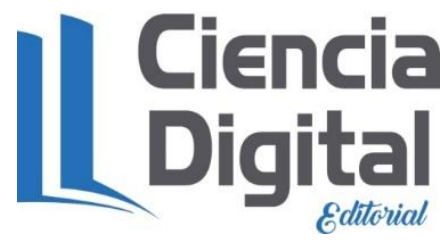

El artículo que se publica es de exclusiva responsabilidad de los autores y no necesariamente reflejan el pensamiento de la Revista Visionario Digital.

El artículo queda en propiedad de la revista y, por tanto, su publicación parcial y/o total en otro medio tiene que ser autorizado por el director de la Revista Visionario Digital.
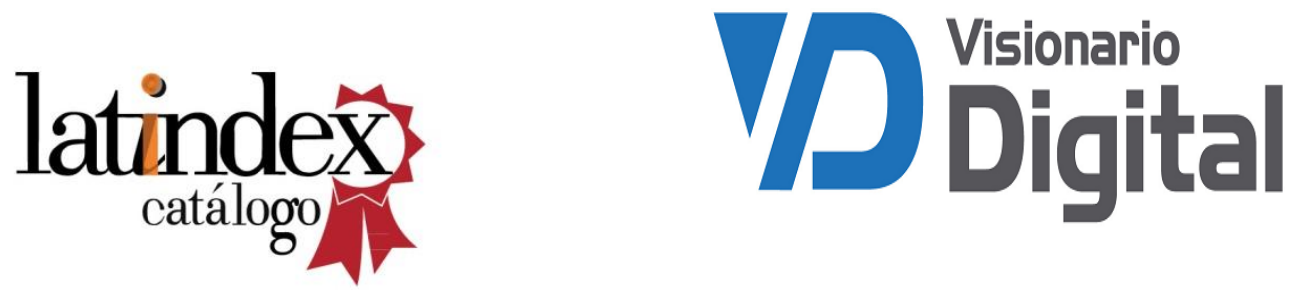